\title{
Toy safety in Singapore: where are we now?
}

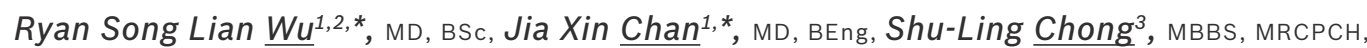 \\ Gene Yong-Kwang Ong ${ }^{3}$, MBBS, MRCPCH, Kee Chong $\mathrm{Ng}^{3}$, MBBS, MMed
}

INTRODUCTION Toy-related injuries, a common reason for emergency department visits in the paediatric population, constitute a significant health burden in Singapore. Although government regulations imposed on toys and childcare-related items are in place, parents and caregivers still play a pivotal role in ensuring toy safety in children. We hypothesised that deficiencies in knowledge surrounding toy safety issues exist in our adult population.

METHODS We conducted a cross-sectional questionnaire study at KK Women's and Children's Hospital, Singapore, to examine the current attitudes, knowledge and practices of the local population regarding toy safety. Hypothetical questions on toy selection were included in the questionnaire to assess respondents' ability to identify appropriate toys and discern potentially dangerous toys for a specified age group.

RESULTS The scores of the 93 respondents showed that they were less able to identify appropriate toys for children in the 1-2 years (66.7\%; 95\% confidence interval [Cl] 61.4\%-72.0\%) and 2-3 years age groups (69.9\%; $95 \% \mathrm{Cl} 65.5 \%-74.3 \%)$ than for children in the $0-1$ year $(82.8 \% ; 95 \% \mathrm{Cl} 79.9 \%-85.7 \%)$ and $3-5$ years age groups (85.2\%; 95\% Cl 81.2\%-89.2\%). Our survey also identified key areas for improvement (e.g. educational efforts and government regulation) in current practices in order to attain a higher level of toy safety.

CONCLUSION Our study highlights the current knowledge deficit in toy safety issues and the need for improvements in regulation, education and surveillance in order to minimise the risk of toy-related injuries in children.

Keywords: emergency, Singapore, survey, toy-related injuries, toy safety

\section{INTRODUCTION}

According to the National SAFE KIDS campaign in the United States (US), approximately 168,000 children aged 14 years and below have visited emergency rooms for toyrelated injuries each year since 2000, and an average of 20 children die from toy-related incidents every year. ${ }^{(1-6)}$ It was estimated that approximately $50 \%$ of these toy-related injuries occurred in children under five years old, with choking being the leading cause of injury among children aged three years and under. $^{(1,7)}$ Although there have been no similar studies conducted in Singapore to date, we opine that toy-related injuries in the paediatric population constitute a significant health burden locally.

Toys that may appear safe - for instance, a balloon - can pose a threat to children and even cause death. In the US, the Child Safety Protection Act requires manufacturers to place labels on packaging for small balls, balloons, marbles and certain toys and games with small parts to warn consumers about choking hazards. ${ }^{(8)}$ In addition, the Federal Hazardous Substances Act ${ }^{(9-11)}$ has banned toys and childcare-related items that pose an electrical, mechanical and/or thermal hazard, as well as products that contain hazardous substances such as lead.

In Singapore, toy safety standards have recently been given a boost by the Standards, Productivity and Innovation Board (SPRING) Singapore, a governmental agency that oversees products and services in Singapore. SPRING Singapore mandates that toys and childcare-related items comply with applicable international, regional or national safety standards. ${ }^{(12)}$ Although governmental regulation offers first-line protection for children from toy-related injuries, parents and caregivers are still primarily responsible for ensuring the safety of their children. Adults can prevent toy-related injuries by being aware of the potential harm that can be caused by certain toys and choosing toys that are safe for their children.

To the best of our knowledge, there is currently no published study on parental knowledge of toy safety and practices in Singapore. We hypothesised that there are deficiencies in the knowledge surrounding toy safety issues among caregivers. Thus, we conducted a survey to examine the current attitudes, knowledge and practices of the Singapore population regarding toy safety and studied the respondents' experiences with toyrelated incidents.

\section{METHODS}

A cross-sectional questionnaire study was conducted from February to April 2012 at the Children's Emergency Department of KK Women's and Children's Hospital $(\mathrm{KKH})$, a tertiary care institution in Singapore with an annual attendance of approximately 158,000 children aged 16 years and below. The study was exempted from review by SingHealth's Centralised Institutional Review Board, Singapore.

${ }^{1}$ Duke-National University of Singapore Graduate Medical School, ${ }^{2}$ Singhealth Pediatrics Residency, Graduate Medical Education Office, ${ }^{3}$ Department of Children's Emergency, KK Women's and Children's Hospital, Singapore

*Both authors contributed equally to the study.

Correspondence: Dr Ryan Song Lian Wu, Singhealth Pediatrics Resident, Graduate Medical Education Office, KK Women's and Children's Hospital, Children's Tower, Level 4, 100 Bukit Timah Road, Singapore 229899. ryan.wu@mohh.com.sg 
A convenience sample was obtained - KKH staff distributed survey forms to accompanying adults of children presenting to the Children's Emergency Department for any medical problems (not restricted to toy-related incidents). Respondents were asked for their demographic information, including age, gender, education, number and age of their children. Respondents were also asked about their concerns and practices regarding toy safety, and their own experiences with toy-related injuries in children. The respondents were given hypothetical questions on toy selection to assess their ability to choose appropriate toys for the stated age groups of children. Pictures of toys were obtained from open sources on the World Wide Web or from personal photography. Based on the recommended guidelines by the US Consumer Product Safety Commission (CPSC) ${ }^{(13)}$ and toy manufacturers, these toys were classified as either safe or potentially dangerous for the specified age groups. All surveys were anonymous, and incomplete surveys or those completed by individuals aged $\leq 16$ years were excluded from analysis.

The questionnaire included a set of hypothetical questions on toy selection for each of the specified age groups: (a) $0-$ 1 year; (b) 1-2 years; (c) 2-3 years; and (d) 3-5 years (refer to Appendix 1, questions 6-9). Each question consisted of 4-6 selections of toys. Out of the 19 possible toy selections, 12 were considered potentially dangerous (boxes marked with a cross in Appendix 1, questions 6-9).

An overall toy-selection score was computed as the percentage of correctly chosen toys over the total number of toys. The overall toy-selection score was then analysed against the background information to identify any factors that might impact the overall toy selection score. The performance of the respondents was further subanalysed using a score that was computed based on the individual questions that applied to the respective age group stratum; this score was termed 'ageappropriate' score. For example, the age-appropriate score for children aged 0-1 year was computed for each respondent by dividing the number of correctly chosen toys in the $0-1$ year age group over the total number of toys in that age group. The mean age-appropriate score for each age group was analysed and compared with the mean scores of the other three age groups. All hypothetical questions were given equal weightage when calculating the scores.

Data was analysed using the GraphPad Prism version 5.0 software (GraphPad Software Inc, San Diego, CA, USA). Unpaired t-test was used to compare the overall toy-selection scores between two subgroups. One-way analysis of variance with Bonferroni multiple comparisons was used to compare the overall toy-selection and age-appropriate scores among multiple subgroups. All tests were two-sided with statistical significance set at $\mathrm{p} \leq 0.05$.

\section{RESULTS}

A total of 107 surveys were collected; of which 14 were excluded from analysis because they were incomplete. Of the
Table I. Additional demographic characteristics of respondents ( $n=93)$.

\begin{tabular}{lc}
\hline Characteristic & No. (\%) \\
\hline Education & \\
Primary & $1(1.1)$ \\
Secondary & $16(17.2)$ \\
Diploma/Junior College & $26(28.0)$ \\
Degree/Masters/PhD & $49(52.7)$ \\
Others & $1(1.1)$ \\
Age group of children* (yrs) & \\
$0-1$ & $19(20.4)$ \\
$1-2$ & $23(24.7)$ \\
$2-3$ & $24(25.8)$ \\
$3-5$ & $29(31.2)$ \\
$>5$ & $30(32.3)$ \\
\hline
\end{tabular}

${ }^{*}$ Children for whom the respondents bought toys; respondents may buy toys for children in more than one age group.

remaining 93 surveys, 55 (59.1\%) respondents were women. The average age of the 93 respondents was $36.3 \pm 7.0$ years and the average age of the respondents' children was 2.9 years. Additional demographic information of the respondents is shown in Table I.

Out of the 93 respondents, 59 (63.4\%) reported being the primary caregiver of their child. Of the remaining 34 respondents, $23(24.7 \%)$ indicated that the primary caregivers of their child were grandparents and domestic helpers. Only 19 (20.4\%) respondents had jobs involving taking care of and supervising children during play. Comparison of the baseline characteristics of the respondents and their mean overall toy-selection scores indicated that factors such as gender $(p=0.495)$, education $(p=0.450)$, being a caregiver $(p=0.559)$ and having a job that involves taking care of or working with children $(p=0.840)$ were not associated with the overall toy-selection scores.

The respondents' ability to select appropriate toys for children of the respective age groups was examined using the age-appropriate scores. The mean scores of the respondents were $82.8 \%$ (95\% confidence interval [CI] $79.9 \%-85.7 \%)$ and $85.2 \%(95 \% \mathrm{Cl} 81.2 \%-89.2 \%)$ for the 0-1 year and 3-5 years age groups, respectively. In contrast, the respondents scored an average of $66.7 \%(95 \% \mathrm{Cl}$ $61.4 \%-72.0 \%)$ and $69.9 \%(95 \% \mathrm{Cl} 65.5 \%-74.3 \%)$ for the $1-2$ years and $2-3$ years age groups, respectively.

Safety was a primary concern among $92.5 \%$ of the respondents when buying a new toy for a child. Other considerations included educational value (83.9\%) and age-appropriateness $(79.6 \%)$. The top three safety aspects that respondents were concerned with were sharp or breakable parts $(93.5 \%)$, swallowing or choking hazard (91.4\%) and the presence of lead paint, chemicals or plastics (68.8\%).

With regard to the practices adopted by our local population, $72.0 \%$ of respondents reported that they refer to the age group listings on toys as a guide before selecting a toy for a child. In contrast, only $4.3 \%$ of respondents seldom or never engaged in this practice. Our study also showed that the majority of respondents $(81.7 \%)$ ensured that the toy was appropriate for 
a child's age before selecting the toy for a child. $63.4 \%$ of the respondents also indicated that they read the safety labels and followed the instructions for use. However, $92.5 \%$ of the respondents indicated that they had previously purchased a toy not meant for a child's age group. Of these respondents, the main reasons for doing so included the caregiver's belief in the toy's educational benefit for the child (50.5\%) and the caregiver's assumption that the child had met the appropriate developmental milestone and was able to play with the toy safely $(41.4 \%)$.

Our study also revealed that $87.1 \%$ of the respondents had experienced toy-related incidents with their children on at least one occasion. The majority of respondents reported that their children had broken off pieces from their toys on at least one occasion $(80.4 \%)$ and/or placed a toy part into their ear, nose or throat $(51.1 \%)$. In addition, $37.0 \%$ of respondents also reported that there had been at least one incident in which their children had been physically injured while playing with a toy. The top reasons cited for these toy-related incidents were wrong use of toys by the child $(44.1 \%)$ and a lack of supervision by caregivers (37.6\%).

\section{DISCUSSION}

Our study revealed that the majority of our respondents (87.1\%) had previously experienced some form of toy-related incident with their child. In addition, more than $50 \%$ of the respondents reported that their children had, on at least one occasion, placed a toy (or its parts) in the ear, nose or throat, consistent with the observation that cases of foreign bodies in the ear, nose and throat are commonly seen in the emergency rooms. ${ }^{(14)}$ In our study, the lack of supervision and wrong use of toys by the child were the most frequently cited reasons for toyrelated injuries. These findings signify that a large percentage of children are at risk for severe toy-related injuries, reiterating the need for an emphasis on toy safety and concerted educational efforts to reduce such risks. Parents and caregivers need to be more involved in their child's play and be aware of any potential harm a toy may pose to their child. Parents can also take on a more proactive role by constantly supervising their child during play and making sure that toys are being used in the correct manner. They should regularly check old and new toys for broken parts, chipped paint, damage and potential danger. Damaged toys should be removed or repaired immediately.

Our respondents' mean overall toy-selection score of $75.8 \%$ suggests that most caregivers were able to select appropriate toys for children of the various age groups. However, when the age-appropriate scores were compared among the various age groups, the respondents did not perform as well when selecting age-appropriate toys for children from the $1-2$ years $(p<0.05)$ and $2-3$ years $(p<0.05)$ age groups as compared to selecting age-appropriate toys for children from the $0-1$ year and 3-5 years age groups. This could be attributed to the fact that the former age groups coincide with the period when children begin to walk and further develop their fine motor skills, while not yet being able to identify potential dangers posed by inappropriate toys. ${ }^{(15,16)}$ Thus, caregivers who are unfamiliar with these developmental milestones are less able to discern between a relatively safe toy and a potentially dangerous one for their child. Conversely, caregivers are generally more wary and careful of newborns because of their vulnerability, and thus performed better at identifying the age-appropriate and potentially dangerous toys in the $0-1$ year age group. For children aged 3-5 years, we postulate that caregivers would have had more experience supervising their child at play with toys and hence were more able to select appropriate toys for them. Moreover, children in the 3-5 years age group are also more mature than those in the younger age groups and would probably be able to identify some of the potential dangers; hence, they are less likely to be involved in toy-related injuries.

Our study also considered the attitudes of caregivers with regard to toys. With safety being one of their primary concerns, respondents considered sharp or breakable parts, swallowing or choking hazard and the presence of lead paint, plastics or chemicals as their top three considerations when selecting a toy. This coincided with the findings of a report in 2010, which identified lead, toxic phthalates, choking and strangulation as major toy hazards. ${ }^{(17)}$ We found that the majority of our respondents ensured that toys were safe for their child by reviewing the age appropriateness of toys, reading safety labels and following the instructions for use. These are good practices that can help to lower the incidence of toy-related injuries and should be encouraged.

However, although $72.0 \%$ of respondents checked the labels for age recommendation, $50.5 \%$ of them bought toys that were not meant for a child's age group because they believed that the toy would benefit the child educationally. Likewise, $41.9 \%$ bought toys that were inappropriate for their child's age, as they believed that their child was developmentally ready to play safely with the toy. It is important that parents and caregivers understand that age recommendation labels are not based on the level of difficulty of a toy for a child of a certain age group, but on the fact that the toy may contain small parts or present dangers to younger children. Hence, we strongly recommend that educational efforts in promoting toy safety be directed at reinforcing age recommendations for all toys so as to avoid unnecessary toy-related injuries.

The strength of our study lies in the wide variety of toys used in our survey for hypothetical toy selection. We followed closely the guidelines from the US CPSC and toy manufacturers when creating the set of questions on hypothetical toy selection as a surrogate for assessment of adults' knowledge on appropriate toy selection. One limitation of the study was the lack of a standardised method of assessing the ability of respondents in choosing the appropriate toys for a child. Other limitations include the different proportions of questions regarding 
potentially dangerous and safe toys within each age group, the lack of clarity of the pictures, especially for toys with small parts, and the study's small sample size. Moreover, with convenience sampling, selection bias could also be present.

The induction of SPRING Singapore has increased the level of toy safety in Singapore through market surveillance and investigation of accidents, incidents or complaints pertaining to controlled goods, including toys. With government regulations in place, parents and caregivers are more likely to be assured that toys sold in Singapore are safe. In the US, the CPSC enforces third party testing and certification of compliance for testing of manufactured toys. ${ }^{(18)}$ Similarly, the Directive 2009/48/EC of the European Parliament and the council of 18 June 2009 require importers and manufacturers to carry out sample testing to protect the health and safety of consumers. ${ }^{(19)}$ One suggestion would be for SPRING Singapore to adopt similar strategies in governing toy safety, including stricter licensing, certification and audits. There should also be stricter regulation on toy safety labelling using predetermined recognised standards set by SPRING Singapore, rather than relying solely on manufacturers' labels.

Meanwhile, we should also focus our educational efforts on those most directly responsible for the safety of Singaporean children. The Health Promotion Board of Singapore, a statutory board whose role is to be the main driver for national health promotion, has a wealth of information and guidelines on toy safety. ${ }^{(20)}$ In addition to increasing awareness on the availability of such information, we also suggest that educational pamphlets and public seminars be conducted to increase outreach efforts, with a focus on parents and caregivers of younger children, so as to better address the current knowledge deficit. A third approach could be through physicians who come into first-line contact with caregivers. Paediatricians and family physicians must be familiar with toy safety issues and should carry out opportunistic education during regular reviews, particularly for those in the high-risk groups (1-2 years and 2-3 years). Finally, as there is limited research on the incidence of toyrelated injuries in Singapore, more studies could be conducted to further evaluate toy safety knowledge in our local population. This information could be used to assist regulatory bodies in addressing any deficiencies, as well as assess the efficacy of implemented strategies by trending the incidence of toy-related injuries over a time period.

In summary, toy-related injuries, which form a significant proportion of visits to the Children's Emergency Department, are easily preventable. It is vital that measures are put in place to ensure the safety of toys for children. Although government regulations play an important role in toy safety, it is vital that parents and caregivers take on a more proactive role in ensuring their child's safety. Our study has demonstrated the existence of knowledge deficits among caregivers, especially when pertaining to children in the younger age groups. We suggest a three-pronged approach through regulation, education and surveillance to increase public awareness and reduce the incidence of toy-related injuries. Together with concerted efforts between multiple agencies and caregivers, we can prevent toy-related injuries and minimise the rate of toy-related incidents, ensuring safe play for young children.

\section{REFERENCES}

1. Tu Y. Toy-related deaths and injuries - calendar year 2009. US Consumer Product Safety Commission [online]. Available at: www.cpsc.gov/library/ toymemo09.pdf. Accessed July 12, 2012.

2. Chowdhury RT. Toy-related deaths and injuries - calendar year 2006. US Consumer Product Safety Commission [online]. Available at: www.cpsc. gov/library/toymemo06.pdf. Accessed July 12, 2012.

3. McDonald J. Memorandum: Toy-related deaths and injuries - calendar year 2003. US Consumer Product Safety Commission [online]. Available at: www.cpsc.gov/library/foia/foia05/os/toymemo03.pdf. Accessed July $12,2012$.

4. McDonald J. Memorandum: Toy-related deaths and injuries - calendar year 2002. US Consumer Product Safety Commission [online]. Available at: www.cpsc.gov/library/foia/foia04/os/toydeath.pdf. Accessed July 12, 2012.

5. McDonald J. Memorandum: Toy-related deaths and injuries - calendar year 2001. US Consumer Product Safety Commission [online]. Available at: www.cpsc.gov/library/toydth01.pdf. Accessed July 12, 2012.

6. McDonald J. Memorandum: Toy-related deaths and injuries, calendar year 2000. US Consumer Product Safety Commission [online]. Available at: www.cpsc.gov/library/toydth00.pdf. Accessed July 12, 2012.

7. Committee on Injury, Violence, and Poison Prevention. Prevention of choking among children. Pediatrics 2010; 125:601-7.

8. For Kids' Sake: Think Toy Safety. In: US Consumer Product Safety Commission Publication 281 [online]. Available at: www.cpsc.gov/ cpscpub/pubs/281.pdf. Accessed July 12, 2012.

9. Federal Hazardous Substances Act of 2011, Pub. L. No. 86-613; 74 Stat. 372 (July 12, 1960).

10. Standard Consumer Safety Specification on Toy Safety. In: American Society for Testing and Materials [online]. Available at: www.astm.org/ Standards/F963. Accessed July 12, 2012.

11. Requirements under the Federal Hazardous Substances Act: Labeling and Banning Requirements for Chemicals and Other Hazardous Substances, 15 U.S.C. § 1261 and 16 C.F.R. Part 1500. In: US Consumer Product Safety Commission [online]. Available at: http://www.cpsc.gov/businfo/ regsumfhsa.pdf. Accessed July 12, 2012.

12. Consumer Protection (Consumer Goods Safety Requirements) Regulations 2011 (CGSR). In: SPRING Singapore [online]. Available at: www.spring.gov. sg/QualityStandards/CPS/Documents/CGSR_InfoBooklet.pdf. Accessed July 12, 2012.

13. Goodson B, Bronson M. Which Toy for Which Child: a Consumer's Guide for Selecting Suitable Toys. US Consumer Product Safety Commission [online]. Available at: http://www.cpsc.gov/cpscpub/pubs/285.pdf. Accessed July 12, 2012

14. Afolabi OA, Alabi BS, Segun-Busari S, Dunmade AD, Foluwasayo E. Paediatric aural foreign bodies: a challenge to care givers. Internet J Otorhinolaryngol 2009; 11:7.

15. Mukherjee A, Haldar D, Dutta S, et al. Ear, nose and throat foreign bodies in children: a search for socio-demographic correlates. Int J Pediatr Otorhinolaryngol 2011; 75:510-2.

16. Figueiredo RR, Azevedo AA, Kós AO, Tomita S. Complications of ENT foreign bodies: a retrospective study. Braz J Otorhinolaryngol 2008; 74:7-15.

17. Trouble in Toyland - 25th Annual Survey of Toy Safety. US Public Interest Research Group [online]. Available at: www.uspirg.org/sites/pirg/files/ reports/toylandbody2010UPDATE.pdf. Accessed July 12, 2012.

18. Toy Safety. In: US Consumer Product Safety Commission [online]. Available at: http://www.cpsc.gov/info/toysafety/index.html. Accessed July 12, 2012.

19. Directive 2009/48/EC of the European Parliament and of the Council of 18 June 2009 on the safety of toys. Official Journal of the European Union [serial online] 2009; L170:1-37. Available at: http://ec.europa.eu/enterprise/ sectors/toys/files/tsd-guidance/tsd_rev_1-6_explanatory_guidance_ document_en.pdf. Accessed October 23, 2013.

20. Health Promotion Board. Home toy safety [online]. Available at: www. hpb.gov.sg/HOPPortal/article?id=3232. Accessed July 12, 2012. 


\section{APPENDIX}

\section{Knowledge, attitudes and practices of toy safety amongst parents/caregivers}

This survey is designed to gather information on the knowledge, attitudes and practices of parents/caregivers in the area of toy safety. Information that you give is anonymous and will help us to understand the level of public awareness of toy safety in Singapore. Thank you for your time and participation in this survey.

1. Background information
$\square$ Male
Female
Age:
Number of children:
Age of children:

2. What is your highest attained level of education?
$\square$ Primary
Secondary
Diploma/Junior College
Degree/Masters/PhD
Others:

3. Which age group of children do you buy toys for? (You can tick more than one box)
$\square$ 0-1 year
$1-2$ years
$\square$ 2-3 years
$\square 3-5$ years
$>5$ years

4. Who spends the MOST time supervising the child during play?
$\square$ Yourself
Others:

5. Does your job involve working closely with children (i.e. taking care of or supervising children during play)?
$\square$ Yes
No

For the following questions (6-9), put a tick in the box below the toy if you think it is safe for a child of the respective age groups (with minimal adult supervision). You can tick more than one box.

6. For a young infant aged $0-1$ year old

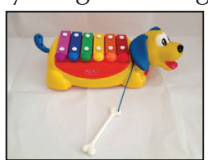

区

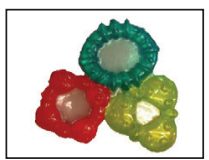

$\square$

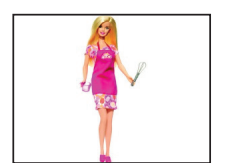

凶

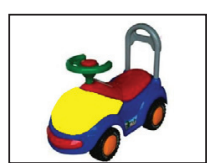

Х

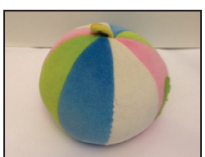

$\square$

7. For children aged $1-2$ years old

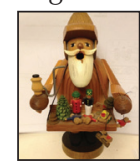

$\mathrm{X}$

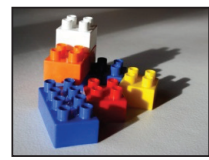

区

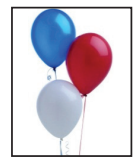

凶

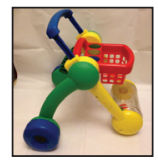

$\square$

8. For children aged $2-3$ years old

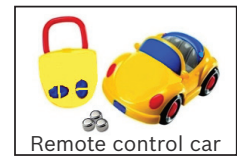

区

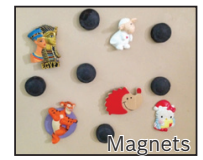

凶

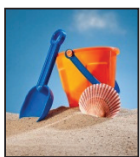

$\square$

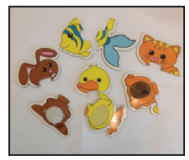

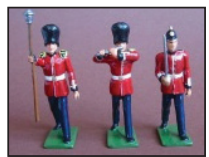

凶

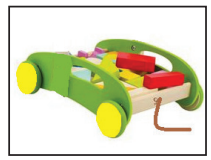

$\square$

9. For children aged $3-5$ years old

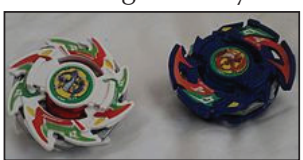

$\mathrm{X}$

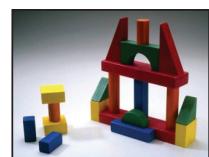

$\square$

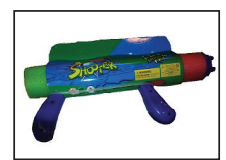

冈

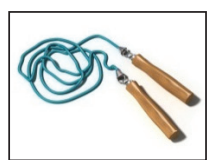

$\mathrm{X}$

10. Tick your TOP 3 considerations when buying a new toy for a child.

$\square$ Child demands for toy

$\square$ Safety

$\square$ Educational

$\square$ Appropriate for child's age

$\square$ Cost

$\square$ Others: 


\section{APPENDIX}

\section{Knowledge, attitudes and practices of toy safety amongst parents/caregivers - continued}

11. Tick your TOP 3 aspects of toy safety you are most concerned with when buying a new toy for a child.

$\square$ Lead Paint / Chemicals / Plastics

$\square$ Swallowing / Choking hazards

$\square$ Sharps / Breakable parts

$\square$ Propelled / Projected objects

$\square$ Electric toys

$\square$ Other:

12. How often do you refer to the age group listed on toys as a guide for how appropriate the toy is for a child?
Always/Most of the time
Sometimes
Never/Seldom

13. If you have ever bought a toy that is not meant for a child's age group, what was the reason for it? (You can tick more than one box)

$\square$ You did not know that the toy was not meant for the child's age group.

$\square$ The child had asked for it or was making a big fuss about the toy.

$\square$ You thought the child would benefit educationally from it.

$\square$ Based on your child's milestones, he/she would be able to play with the toy safely.

$\square$ The toy was shared with other siblings.

$\square$ Others:

14. What do you do before selecting a toy for a child? (You can tick more than one box)

$\square$ Check if the toy is being recalled or banned.

$\square$ Read safety labels carefully and follow instructions for use.

$\square$ Ensuring the toys are appropriate for the child's age group.

$\square$ Nothing, toys in Singapore are generally safe.

15. In your experience with children, how often have the following events occurred? $1=$ Never, 2 = Sometimes, $3=$ More than five times in a year

Child swallowed toy object.

Child placed toy object in ear, eyes or nose.

Child was physically injured by toy object.

Child broke off pieces of toys.

Child chewed off paint from toy.

Child was electric shocked by toy.

Others:

$\begin{array}{lll}1 & 2 & 3 \\ 1 & 2 & 3 \\ 1 & 2 & 3 \\ 1 & 2 & 3 \\ 1 & 2 & 3 \\ 1 & 2 & 3 \\ 1 & 2 & 3\end{array}$

16. If you have circled 2 or 3 in any of the options available in question 15, what do you think is the most likely reason that could have contributed to the occurrence of the abovementioned events?

$\square$ Lack of supervision by caregivers.

$\square$ Toys were recommended for use in older children.

$\square$ Toys were not maintained or stored properly.

$\square$ Child was using the toy in the wrong way.

$\square$ Caregivers were not aware of the safety issues with the toys.

$\square$ Others:

Comments (if any): 